\title{
A Single Intravenous Injection of Oxycodone Hydrochloride In The Treatment of Acute Post-operative Pain after Laparoscopic Rectal Tumor Resection
}

\author{
Chunlu Zhang ${ }^{1}$, Jiali Ding ${ }^{1}$, Bin Wang ${ }^{1}$ and Peng Chen ${ }^{1, a}$ \\ ${ }^{1}$ Department of Anesthesiology, China-Japan Union Hospital, Ji Lin University, Changchun, China
}

\begin{abstract}
Objective: To investigate the efficacy of a single intravenous injection of oxycodone hydrochloride in patients undergoing laparoscopic rectal tumor resection with acute postoperative pain and evaluate the quality of Anesthesia recovery period. Methods:60 patients for laparoscopic rectal tumor resection, were randomly divided into two groups $(\mathrm{n}=30$ each): oxycodone hydrochloride injection group (group I) and fentanil injection group (group II). oxycodone $0.05 \mathrm{mg} / \mathrm{kg}$ (saline dilution of $1 \mathrm{mg} / \mathrm{ml}$ ) or fentanil $0.1 \mathrm{ug} / \mathrm{kg}$ was injected intravenously at the end of surgery. Assessing the OAA / S sedation grade and Prince-Henry postoperative pain scores. Results: Demographic data and anesthesia characteristics were similar in the two groups. Group II's Prince-Henry pain score was significantly higher than the group I's ( $\mathrm{P}<0.01$ ). Conclusion: xycodone injection can reduce the incidence of acute pain after Laparoscopic rectal tumor resection, and effectively relieve the occurred acute pain, the clinical use of oxcodone is safe and effective.
\end{abstract}

\section{Background}

At present, the proportion of laparoscopic surgery in the surgical operation increased year by year because of its small trauma and fast recovery. The pain after laparoscopic operation has aroused great attention. The pain of visceral and radiation were more obvious after laparoscopic surgery in addition to the pain of operative incision by stretching stimulation of the diaphragmatic peritoneum and artificial carbon dioxide pneumoperitoneum and position and so on. Most clinical use of analgesics is opioid now ${ }^{[1]}$. However, it is limiting use in the recovery period because of the respiratory depression and other side effects of opioid analgesics. Oxycodone, a potent semi synthetic opioid drugs, is a type of double receptor agonist of opioid ( $\mu$ receptor and $\kappa$ receptor), and is widely used in moderating to severe cancer pain, postoperative acute pain and neuropathic pain ${ }^{[2]}$. The pharmacological effects of oxycodone is similar with morphine ${ }^{[3]}$. The analgesic effect of visceral pain of oxycodone is better than other opioid drug. The prominent advantage is that the sedation and respiratory depression

\footnotetext{
${ }^{a}$ Corresponding author: chenpeng5481@sina.com
} 
is mild. The study that the analgesic effect of oxycodone injection on the recovery period of acute pain after laparoscopic surgery is not too much. The control effect, the quality of recovery and other side effects after a single intravenous injection of oxycodone hydrochloride and fentanyl in patients with acute pain recovery period of laparoscopic resection of rectal tumor at the end of surgery are compared in this experiment. It can provide reference for clinical work.

\section{Materials and methods}

\subsection{Clinical data}

This study was approved by the Ethics Committee of our hospital and signed informed consents with patients. Select 60 patients who need tumor resection by laparoscopic rectal. ASA classI - class II. Male 26 cases, female 34 cases, aged 40-76 years old. No chronic pain and analgesic or sedative. No long-term history of antidepressants. No alcohol abuse history. No use of sedatives and antiemetic and anti allergic drugs for $24 \mathrm{~h}$ before operation. Patients were divided into two group randomly by the random number table method:Oxycodone hydrochloride injection group (group I) and fentanyl injection group (Group II).

\subsection{Anesthesia methods}

Opened venous pathway, monitored of blood pressure (BP), heart rate (HR), blood oxygen saturation $\left(\mathrm{SpO}_{2}\right)$, electrocardiogram (ECG) when the patients went into the operating room routinely. Intravenous injection midazolan $0.03 \mathrm{mg} / \mathrm{kg}$, propofol $1 \sim 2 \mathrm{mg} / \mathrm{kg}$, sufentanil $0.5 \mathrm{ug} / \mathrm{kg}$, vecuronium $0.1 \mathrm{mg} / \mathrm{kg}$ was used in anesthesia induction. Inserted the endotracheal tube after aerobic nitrogen removal,and mechanical ventilation. Experimented expiration-end gas monitoring,maintaining expiration-end $\mathrm{CO}_{2}$ (PetCO2) $35-45 \mathrm{mmHg}$. The anesthesia was maintained with total inhalation. Inhaled sevoflurane. Maintained the depth of anesthesia in $1.2 \sim 1.5 \mathrm{MAC}$, which no additional sufentanil during the operation. Intravenous injection of vecuronium $0.05 \mathrm{mg} / \mathrm{kg}$ when necessary. Stoped sevoflurane, adjusted the oxygen flow rate, eluted sevoflurane rapidly and gived oxycodone injection $0.1 \mathrm{mg} / \mathrm{kg}$ or fentanyl injection $1 \mathrm{ug} / \mathrm{kg}$ at the end of the operation. When the MAC were below 0.2 , call patients per minutes. Pulled out the tracheal tubes after the patients were clear, the reflex activity were recovered. All the patients in each group were treated by analgesia pump after extubation.

\subsection{Monitoring index}

The time of anesthesia, operation, recovery, extubation and were recorded. The blood pressure, heart rate, $\mathrm{SpO}_{2}$ of the moment of operation completing and extubation at $10 \mathrm{~min}, 20 \mathrm{~min}, 15 \mathrm{~min}$, and $5 \mathrm{~min}$ after extubation were recorded. Each OAA/S was assessed with a $5 \mathrm{~min}$ sedation scale after extubation. ( Level 5, have a swift response to normal voice. Level 4, indifferent of normal voic. Level 3, only response to calling for name loudly or repeatedly. Level 2, only response to a slight shake on the shoulder or head. Level 1, no response to mild pushing and shaking. Level 0 , to squeeze trapezius muscle. OAA/S further divided into excessive sedation score 2 or less). Postoperative pain Prince Henry scores.( 0 points, no pain when coughing. 1 points, pain when coughing. 2 points, the depth of breathing when there is pain, quiet, no pain. 3 points, the rest of the state that there is pain, but more light, can endure. 4 points, resting state that there is severe pain, unbearable. The Prince-Henry score is greater than or equal to 3 divided into the recovery period of acute pain.). The occurrence of agitation, nausea, vomiting, respiratory depression and other adverse reactions were recorded. 


\subsection{Statistical Analysis}

Using statistical software SPSS 17.0 for data processing. The measurement data of normal distribution are expressed as mean plus or minus standard deviation $(\bar{\chi} \pm \mathrm{s})$. Variance analysis between groups was compared using $\mathrm{t}$ test. The analysis of variance of repeated measurement was used in counting data. $\mathrm{P}$ $<0.05$ for the difference was statistically significant.

\section{Result}

A. There were no significant differences in sex, age, body weight and BMI status between two groups, $\mathrm{P}>0.05$. See Table 1 .

Table 1. Comparison of two groups of patients in general $\left(\mathbf{n}=\mathbf{3 0}, \chi^{ \pm s}\right)$

\begin{tabular}{cccccc}
\hline group & $\begin{array}{c}\text { The number } \\
\text { of cases }\end{array}$ & $\begin{array}{c}\text { Male } / \\
\text { female }\end{array}$ & Age(n) & Weight $(\mathrm{kg})$ & BMI \\
\hline group I & 30 & $12 / 18$ & $55.17 \pm 8.59$ & $59.40 \pm 8.10$ & $22.55 \pm 2.27$ \\
group II & 30 & $14 / 16$ & $55.97 \pm 9.78$ & $58.53 \pm 6.97$ & $24.07 \pm 2.85$
\end{tabular}

B. Comparison of anesthesia time and operation time between two groups, No statistical difference, $\mathrm{P}>0.05$.See Table 2 .

Table 2. Comparison of anesthesia time and operation time between two groups

\begin{tabular}{ccc}
\hline group & anesthesia time $(h)$ & operation time $(h)$ \\
\hline group I & $4.00 \pm 0.50$ & $4.29 \pm 0.50$ \\
group II & $3.83 \pm 0.33$ & $4.20 \pm 0.35$
\end{tabular}

C. The spontaneous breathing recovery time, extubation time and recovery time of Fentanyl group was longer than oxycodone group. The difference was statistically significant $(p<0.05)$. See Table 3.

Table 3. The spontaneous breathing recovery time, extubation time and recovery time of Fentanyl group

\begin{tabular}{cccc}
\hline group & $\begin{array}{c}\text { The spontaneous } \\
\text { breathing } \\
\text { recovery time } \\
(\mathrm{min})\end{array}$ & $\begin{array}{c}\text { extubation time } \\
(\mathrm{min})\end{array}$ & $\begin{array}{c}\text { recovery time } \\
(\mathrm{min})\end{array}$ \\
\hline group I & $7.57 \pm 3.22$ & $13.00 \pm 4.09$ & $14.80 \pm 5.22$ \\
group II & $12.87 \pm 3.18$ & $18.77 \pm 4.23$ & $19.80 \pm 4.47$ \\
\hline
\end{tabular}


D. Repeated measure analysis of variance indicate that the change trend of heart rate and mean arterial pressure of oxycodone group is same with the Fentanyl group at 20 minutes after extubation. See Figure 1,2.

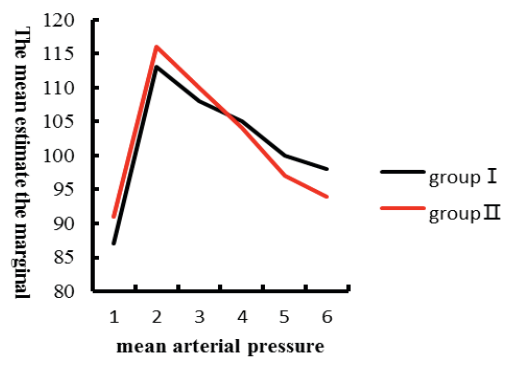

Figure 1. Contrast figure of mean arterial pressure

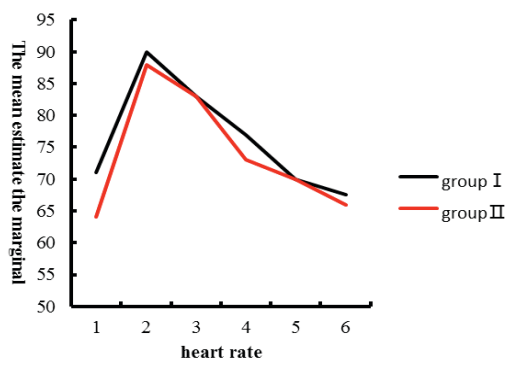

Figure 2. Contrast figure of heart rate

E. Blood oxygen saturation monitoring data displayed that oxygen saturation in fentanyl group decreased more significantly than that of oxycodone group. When the blood oxygen saturation data is lower than $90 \%$, the oxygen mask is required. The number of cases in oxycodone group is one while the number in fentanyl group is five. See Figure 3.

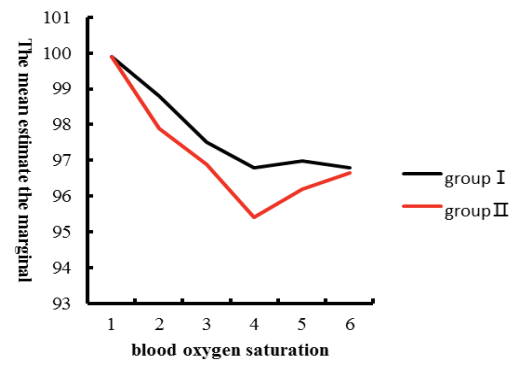

Figure 3. Contrast figure of blood oxygen saturation

F. OAA/S sedation score showed fentanyl group at each time point was lower than that of oxycodone group. The difference was statistically significant $(\mathrm{p}<0.05)$. See Figure 4. 


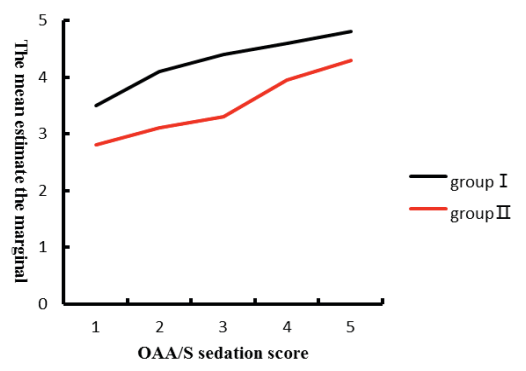

Figure 4. Contrast figure of OAA/S sedation score

G. Prince-Henry pain scores showed the score of fentanyl group was higher than oxycodone group. The difference was statistically significant $(p<0.05)$. See Figure 5 .There were 2 patients in anesthesia recovery period having acute postoperative pain in oxycodone group of experiment. Fentanyl group had 8 patients with acute pain after surgery. When the Prince-Henry score was more than 3 points, emergency analgesia was required. The symptoms would be improved obviously after intravenous oxycodone $(0.1 \mathrm{mg} / \mathrm{kg})$.

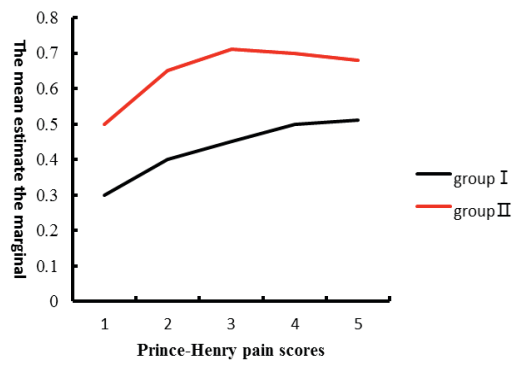

Figure 5. Contrast figure of Prince-Henry pain scores

H. There was no significant difference in the side effects of nausea and vomiting between two groups.

\section{Discussion}

Acute pain after operation in patients can cause a series of complications such as high blood pressure, increased heart rate, agitation, respiratory depression and so on. The characteristic of acute pain after laparoscopic operation is from visceral and radiation in addition to surgical incision. Because the pain occurs in the recovery period, the postoperative analgesia pumps and other conventional analgesia methods can not very well play the analgesic effection. It is the original intention of this study that how to do a good job of convergence and the patient is truly painless. At present, opioid drugs such as morphine, fentanyl, sufentanil is usually used in the recovery period of analgesia. However, this kind of drugs would cause respiratory depression, drowsiness and other complications with the increased of dose. Therefore, because of the quality of the patient's recovery and the fear of respiratory depression, the treatment of the recovery period is often not satisfactory. Oxycodone injection, is a new type of double opioid receptor agonist. In theory, there is a good curative effect on cutting pain and visceral pain. The effect of respiratory depression was mild and no obvious sedation. The purpose of this experiment was to compare the effects of acute pain and the quality of recovery in a single intravenous injection of oxycodone hydrochloride and fentanyl in laparoscopic resection of rectal tumor. 
In the experiment, the Prince-Henry scores of oxycodone group were lower than fentanyl group at each time point. The number of cases in oxycodone group that occurred acute postoperative pain was less than fentanyl group. There are three possible reasons.

1. In the course of laparoscopic rectal tumor resection, by the stretching stimulation of the diaphragmatic peritoneum and artificial carbon dioxide pneumoperitoneum and position and other factors, visceral and radiation pain were more obvious after laparoscopic surgery in addition to the operative incision pain. Some studies have found that $63 \%$ of patients under the diaphragm and shoulder pain after laparoscopic surgery, and the degree and duration of pain is often more than operative incision pain. Patients with $73 \%-80 \%$ after laparoscopic surgery need to use analgesics to relieve acute pain after surgery. The onset time of fentanyl and oxycodone after intravenous injection is similar (2-3mins) and they were all strong opioids. They are mainly mediated by opioid receptors on the presynaptic membrane of nerve terminals in CNS. These opioid receptors belong to the $G$ protein coupled receptor family $(\mu-, \kappa-$ and $\delta-$ ). Fentanyl is mainly through the $\mu$ receptor activation and the cutting and other sharp pain analgesic effect is good. While oxycodone is double opioid receptor agonist ( $\mu$ receptor and $\kappa$ receptor) and has good effect on acute postoperative pain and nerve pain. The pharmacological effects of oxycodone were similar with morphine ${ }^{[4]}{ }^{5]}$. The oxycodone receptor is $\kappa$ receptor. In particular, the $\kappa 2 b$ receptor can help achieve the purpose of analgesia $^{[6]}$. So the analgesic effect of oxycodone on visceral pain is better than other opioids. Animal experiments also showed that the $\kappa$ receptor $^{[7,8,9]}$ is related to the relief of visceral pain. Therefore, the control of oxycodone on postoperative recovery period of acute pain in visceral pain may be better after laparoscopic resection of rectal tumor ${ }^{[10,11]}$.

2. Because of fentanyl and oxycodone in fat-soluble difference is big, the rapid redistribution of fentanyl after intravenous injection may result in the decrease of analgesic effect.

3. In this experiment, only use the conventional dose sufentanil in induction phase, minimal residual dose at the end of the operation.

OAA/S sedation scale of oxycodone group was higher than that of fentanyl group. The number of excessive sedation was less than that of fentanyl group(1:9). The number of $\mathrm{SpO}_{2} \leq 90 \%$ cases was less than fentanyl group. Spontaneous breathing recovery time, extubation time and recovery time were shorter than in the fentanyl group. There are two possible reasons.

1. Oxycodone play predominate pharmacological effectors mainly through $\kappa$ receptor and the $\mu$ acceptor activation is weak. Therefore,reduce the complications such as drowsiness and respiratory depression that occur at the same time of analgesic aim realizing through the $\mu$ receptor agonist ${ }^{[12,13]}$. Due to the good analgesic effect and the patient's breathing is affected by the pain of small,oxycodone group may be greater rate.

2. Oxycodone play predominate pharmacological effects mainly through a $\kappa \mathrm{r}$ eceptor. The synergistic effect of sevoflurane of oxycodone was weaker than that of fentanyl. Therefore, the influence of the quality of recovery of patients may be more weak than fentanyl group.

In summary, a single intravenous injection of oxycodone $0.1 \mathrm{mg} / \mathrm{kg}$ can prevent the occurrence of acute pain after operation better than intravenous injection of fentanyl $1 \mathrm{ug} / \mathrm{kg}$ for patients of laparoscopic resection with rectal tumors at the end of the operation. Excessive sedation and respiratory depression and other side effects are smaller. The recovery of general anesthesia will be more safe.

\section{References}

1. Sun Zhengyi, Lang Jinghe, Leng Jinhua, pain after laparoscopic surgery. Chinese modern surgery magazine.2000; 11; 30 (10): 309-311.

2. Xu Jianguo Pharmacology and clinical application of oxycodone hydrochloride. Journal of Clinical Oncology, 2014,5,30 (5): 511-513.Kalso E.Oxycodone.J Pain Symptom Manage.2005;29:S47-56.

3. Ross FB,Smith MT.The intrinsic antinociptive effects of oxycodone appear to be kappa-opioid 
receptor mediated.Pain 1997;73:151-157.

4. Nozaki C,Saitoh A,Tammura N,Kamei Jantinociceptive effect of oxycodone in diabetic mice.Eur J Pharmacol 2005;524:75-79.

5. Nielsen C,Ross FB,Lotfipour S,Saini KS,Smith MT.Oxycodone ahd morphine have distinctly different pharmacological profiles:radioligand binding and behavioural studies in two rat models of neuropathic pain.Pain 2007;132:289-300.

6. 2009 years of adult postoperative pain treatment expert consensus, the Chinese Medical Association Branch of anesthesiology.

7. Kalso E.Oxycodone.J Pain Symptom Manage.2005;29:S47-56.

8. Lugo RA,Kern SE.The pharmacokinetics of oxycodone.J Pain Palliat Care Pharmacother 2004;18:17-30.

9. Okura T, Hattori A, Takano Y, et al. Involvement of the pyrilamine transporter, a putative organic cation transporter, in blood-brain barrier transport of oxycodone. Drug Metab Dispos 2008; 36: 2005- 2013.

10. M Kokki,P Valitalo,et al.central nervous system penetration of oxycodone after intravenous and epidural administration.British Journal of Anaesthesia.2014;112(1):133-140.

11. Xu Xing, $\mathrm{Wu}$ Xinmin, Xue Zhang Gang, Wang Xiangrui, Xiong, Tian Ming, Chen Yao. Oxycodone hydrochloride injection for postoperative analgesia efficacy, safety and prospective, randomized, double-blind, multicenter, positive controlled clinical research. Chinese Journal of Anesthesiology, 2013; 3; 33 (3): 269-274.

12. Sun Yang, Lei Jun, Wang Meiqing, Cui Yan. Effect of oxycodone on quality of thoracic surgery with general anesthesia recovery period. Journal of clinical anesthesiology, 2014; 10; 30 (10): 1032-1033. 